\title{
An application of a scanning light-addressable potentiometric sensor for label-free DNA detection
}

\author{
Chunsheng $W_{u^{1,2^{*}}}$, Arshak Poghossian ${ }^{2,3}$, Carl Frederik Werner ${ }^{2}$, Thomas Bronder ${ }^{2}$, Matthias Bäcker ${ }^{2}$, \\ Ping Wang ${ }^{1}$, Michael J. Schöning ${ }^{2,3}$ \\ *cswu@zju.edu.cn \\ ${ }^{1}$ Biosensor National Special Laboratory, Key Laboratory for Biomedical Engineering of Ministry of \\ Education, Department of Biomedical Engineering, Zhejiang University, 310027 Hangzhou, China \\ ${ }^{2}$ Institute of Nano- and Biotechnologies, FH Aachen, Campus Jülich, 52428 Jülich, Germany \\ ${ }^{3}$ Peter Grünberg Institute (PGI-8), Research Centre Jülich GmbH, 52425 Jülich, Germany
}

\begin{abstract}
:
In this study, a scanning light-addressable potentiometric sensor (LAPS) was applied for label-free and direct electrical detection of DNA (deoxyribonucleic acid) hybridization for the first time. The functioning principle of the sensor is based on an electrostatic detection of the intrinsic molecular charge of DNA molecules together with an ion-concentration redistribution within intermolecular spaces. The LAPS signal shows a distinct dependence on the concentration of the complementary target DNA. The mechanism of the shift of the photocurrent-voltage curve and the LAPS signal generation induced by the DNA immobilization and hybridization event is discussed.
\end{abstract}

Key words: label-free detection, DNA hybridization, DNA biosensor, light-addressable potentiometric sensor

\section{Introduction}

DNA (deoxyribonucleic acid) hybridization detection plays a crucial role in many fields such as DNA diagnostics, gene analysis, fast detection of biological warfare agents, and forensic applications [1]. Many optical and electrochemical methods involving labelling of DNA probe or target molecules have been developed for the detection of DNA hybridization, which are, however, usually timeconsuming and costly. Therefore, recently, extensive efforts have been invested to develop various semiconductor field-effect devices (FED) for a fast and label-free electrical detection of DNA molecules by their intrinsic molecular charge [2,3]. FED-based biosensors and DNA chips are of special interest since the fast development of micro- and nanofabrication technologies makes them cheaper, easier for integration and large-scale production. Although FEDs have been widely applied in the development of DNA biosensors, they still greatly suffer from the restricted and discrete measurement sites due to the non-flexible gateelectrode of the individual FED.

On the other hand, the light-addressable potentiometric sensor (LAPS), which is a type of silicon-based FED for surface potential detection, can overcome the limitations mentioned above [4]. The signal from every spot on the LAPS surface can be read out by focusing a modulated irradiation there. Due to some advantages such as the selection of the measurement point and the spatial resolution, LAPS have become popular in many chemical and biological applications [5]. In the recent decade, LAPS has been further developed into a platform that can perform multi-spot measurements [6]. However, it is very little known about label-free DNA detection with the LAPS.

In this work, we present the results of applying the scanning LAPS for a label-free DNAhybridization detection with direct electrical readout for the first time. Single-stranded DNA (ssDNA) probe molecules were covalently immobilized on the silanized LAPS surface. The photocurrent-voltage (I-V) curve shifts of the LAPS chip induced by the intrinsic charge of DNA molecules were detected by measuring the photocurrent on each spot of the LAPS surface with a focused scanning laser. The DNA-hybridization event on the LAPS surface was further validated by fluorescent staining. The possible mechanism of the DNAhybridization signal generation is proposed and discussed. 


\section{Materials and methods}

\subsection{LAPS chip fabrication}

LAPS chips consisting of a $\mathrm{Si}-\mathrm{SiO}_{2}-\mathrm{Ta}_{2} \mathrm{O}_{5}$ structure were fabricated using $\mathrm{p}$-doped silicon wafer with a thickness of $400 \mu \mathrm{m}(<100>, 1-10$ $\Omega \mathrm{cm}$ ). First, a $\mathrm{SiO}_{2}$ layer with thickness of 30 $\mathrm{nm}$ was prepared on the surface of the silicon wafer by thermal oxidation. Then, a $50 \mathrm{~nm}$ thick Ta layer was deposited on the top of the $\mathrm{SiO}_{2}$ layer. The Ta layer was then oxidized in an oxidation oven at a temperature of $520{ }^{\circ} \mathrm{C}$, resulting in a $\mathrm{Ta}_{2} \mathrm{O}_{5}$ layer with a thickness around $60 \mathrm{~nm}$. Finally, a $300 \mathrm{~nm}$ Al layer was deposited on the rear side of the silicon wafer to create an Ohmic contact. The inset in Fig. 1 shows the image of the LAPS chip with a $\mathrm{Ta}_{2} \mathrm{O}_{5}$ gate.

\subsection{DNA immobilization}

All ssDNA sequences including 5'-end aminomodified probe ssDNA (with sequence of $5^{\prime}$ ACCTGGGGGAGTATTGCGGA-3'), mismatch ssDNA (5'-AGGCGTTATGAGGGGGTCCA-3') and complementary target ssDNA (5'TCCGCAATACTCCCCCAGGT-3') were purchased from Eurofins (Eurofins MWG Synthesis $\mathrm{GmbH}$, Ebersberg, Germany).

For the covalent immobilization of 5'-end amino-modified ssDNA probes, the LAPS surface was treated with $0.1 \%(\mathrm{v} / \mathrm{v})$ 3aminopropyltriethoxysilane (APTES) in toluene solution at room temperature (RT) for $1 \mathrm{~h}$ to form a smooth thin film of self-assembled APTES (Fig. 1). After rinsing with toluene and ethanol, the LAPS surface was incubated with $5 \%$ glutaraldehyde for $12 \mathrm{~h}$ at room temperature (RT). Then, the chip surface was rinsed with distilled (DI) water and dried with nitrogen stream. In the next step, the LAPS surface was incubated with $10 \mu \mathrm{M}$ probe
ssDNA solution for $12 \mathrm{~h}$ at RT. ssDNA probes were previously dissolved in $\mathrm{DI}$ water and diluted with PBS solution (0.1 M PBS, $\mathrm{pH}$ 8.5) to achieve the desired concentration of DNA. After incubation, the chip was washed with DI water and incubated with $1 \%$ bovine serum albumin (BSA) solution for $30 \mathrm{~min}$ at RT to block unreacted aldehyde residues and other non-specific binding sites. Finally, the LAPS surface was rinsed with PBS solution and dried with nitrogen stream. The LAPS chip with immobilized probe ssDNA was stored at $4{ }^{\circ} \mathrm{C}$.

\subsection{LAPS measurement setup}

A scanning LAPS setup used in this study is described in detail in our previous report [6]. Fig. 2 shows the schematic diagram of the scanning LAPS measurement setup used in this study. Briefly, the light source is a modulated laser with a wavelength of $780 \mathrm{~nm}$ and a diameter of $70 \mu \mathrm{m}$. A nanomotor (GMN, Germany) was used for a precise (with the minimum stepwise of $40 \mathrm{~nm}$ ) and large area (18 $\mathrm{mm}^{2}$ ) laser scanning of a LAPS surface. For the experiments, a bias voltage ranging from $-2.0 \mathrm{~V}$ to $+2.0 \mathrm{~V}$ is applied between the reference electrode and the rear-side contact of the LAPS, and the I-V curve was recorded in each measurement spot. An $\mathrm{Ag} / \mathrm{AgCl}$ electrode with saturated $\mathrm{KCl}$ was used as reference electrode. The $\mathrm{NI}$ data collection card was employed to collect the data and the customized LabView software was used as the controller of the setup.

Before DNA hybridization, the $I-V$ curve of the LAPS chip was recorded in a measurement solution (0.2 mM PBS, $\mathrm{pH}$ 7.0). Then, the measurement solution in the detection chamber was removed and the LAPS surface was exposed to the DNA-hybridization solution containing the complementary target ssDNA or mismatch ssDNA.

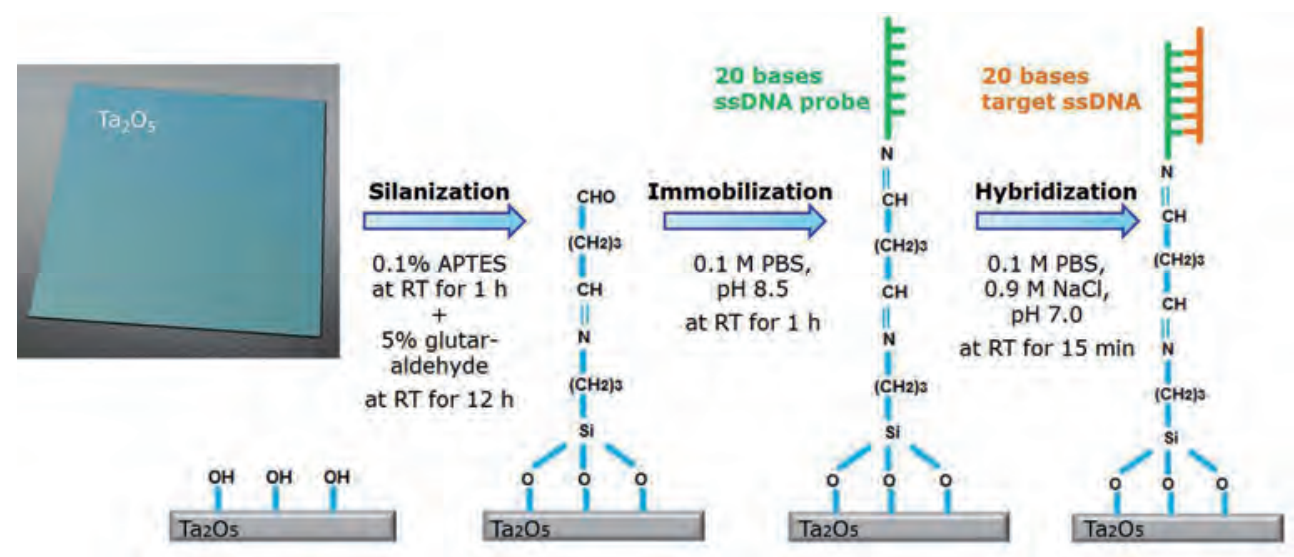

Fig. 1. Image of the LAPS chip with a $\mathrm{Ta}_{2} \mathrm{O}_{5}$ gate and schematic of the covalent immobilization of aminomodified ssDNA probe molecules on a LAPS surface and hybridization with complementary target ssDNA. 


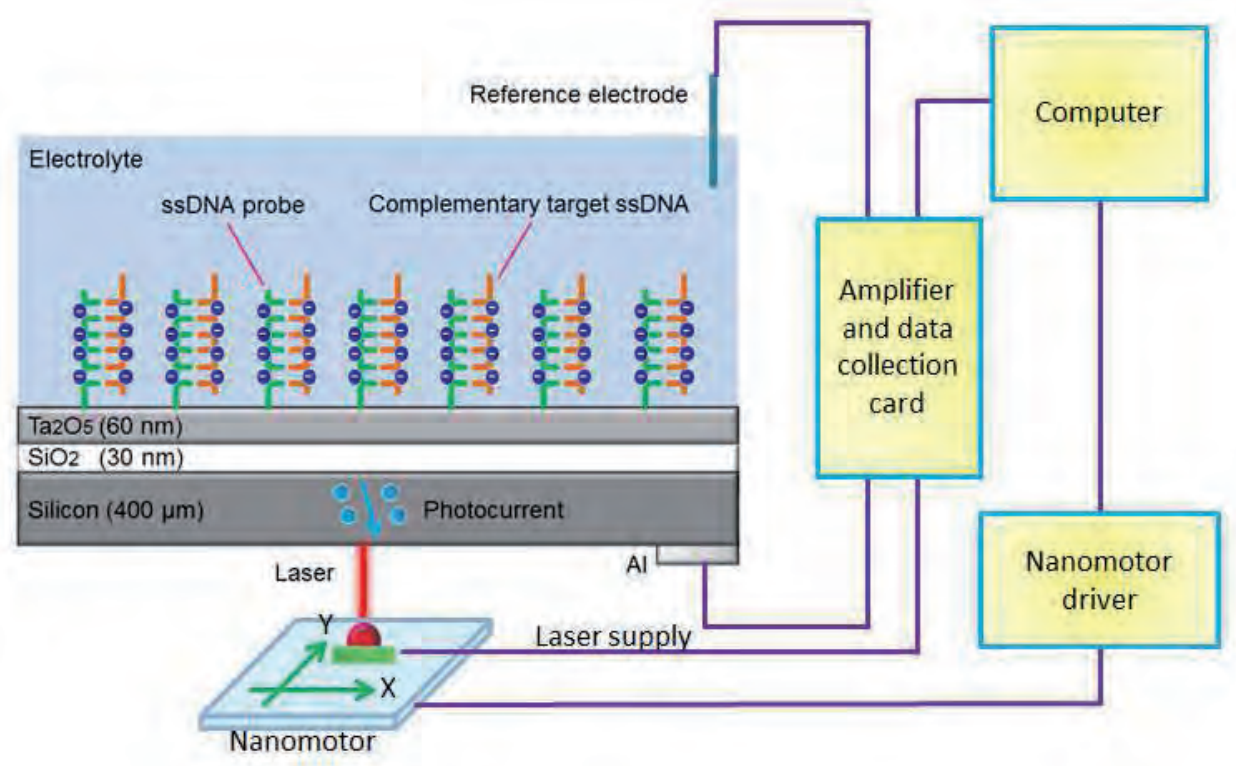

Fig. 2. Schematic diagram of a scanning LAPS measurement setup.

For the preparation of the hybridization solution, complementary target ssDNA and mismatch ssDNA molecules were dissolved in DI water and then, diluted with a buffer solution $(0.1 \mathrm{M}$ PBS, pH 7, with $0.9 \mathrm{M} \mathrm{NaCl}$ ) to achieve the desired concentration of target DNA. After 30 min hybridization at RT, the LAPS surface was washed with the measurement solution to remove the unreacted target ssDNA molecules and then, the $I-V$ curve of the LAPS chip was recorded in the measurement solution again. The shift of the I-V curves (measured before and after the DNA hybridization) along the voltage axis is used as a quantitative indicator to characterize the DNA-hybridization event. All measurements were carried out at RT. The whole setup was shielded with a copper box to exclude ambient light and to minimize the influences of environmental factors to the measurements.

\subsection{Fluorescence microscopy characterization}

In order to test the effectivity of protocols used for the DNA immobilization and hybridization as well as to verify the DNA hybridization on the surface of the LAPS chip, fluorescencemicroscopy measurements were done using a blue-fluorescent dye 4', 6-Diamidino-2phenylindole dihydrochloride (DAPI). The DAPI molecules preferentially bind to doublestranded DNA (dsDNA) (associate with AT clusters) and produces $\sim 20$-fold fluorescence enhancement. The fluorescence microscope used in this study was Axio Scope.A1 (Zeiss, Germany). For the fluorescence microscopy characterization, separate LAPS chips consisting of a p-Si-SiO ${ }_{2}$ structure were functionalized with probe ssDNA molecules and then, hybridized with complementary target ssDNA to form dsDNA according to the protocol described in section 3.2. DAPI staining was performed immediately before the fluorescence microscopy measurements according to the following protocol:

- equilibration of the sensor surface with PBS,

- dilution of a DAPI stock solution to $300 \mathrm{nM}$ in PBS,

- application of $\sim 100 \mu \mathrm{L}$ of this diluted DAPI staining solution to the LAPS surface, incubation for 1-5 min at RT followed by rinsing the sensor surface several times in PBS buffer.

\section{Results and discussion}

$3.1 \mathrm{pH}$ sensitivity of the LAPS and fluorescence measurements

To use the LAPS chip as a transducer for the detection of surface charge changes, it is necessary to check the capability of the prepared chip for photocurrent generation as well as to determine the optimal working parameters such as the threshold of the bias voltage, the modulation frequency and the intensity of the laser beam. For this purpose, the functionality of the LAPS chips was first tested in $\mathrm{pH}$ buffer solutions. The results of $\mathrm{pH}$ measurements (data not shown) indicate that LAPS chips with $\mathrm{Ta}_{2} \mathrm{O}_{5}$ surface exhibit a $\mathrm{pH}$ sensitivity of $56.7 \mathrm{mV} / \mathrm{pH}$ that is in good agreement with results reported for $\mathrm{Ta}_{2} \mathrm{O}_{5}$ layers previously [7-9].

DNA hybridization on the LAPS surface was validated by fluorescent staining using DAPI, which preferentially binds to dsDNA and can generate a significant fluorescence enhancement upon binding with dsDNA. 

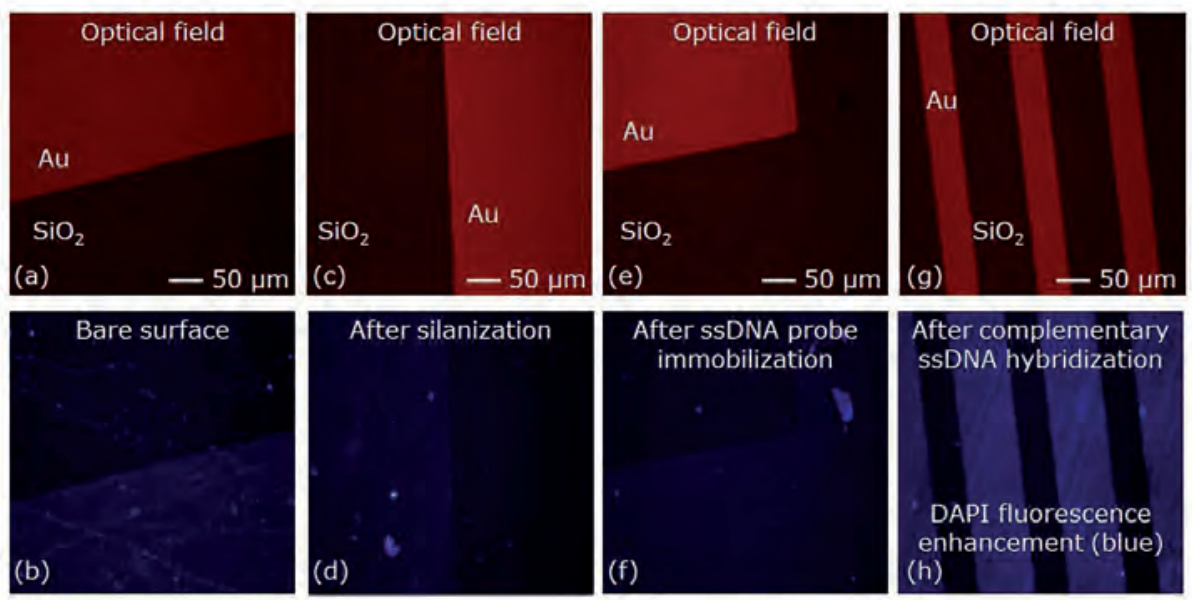

Fig. 3. Results of fluorescent staining with DAPI on various LAPS surfaces. (a) and (b) bare LAPS surface (without any modification); (c) and (d) after silanization; (e) and (f) after ssDNA immobilization; (g) and (h) after hybridization with complementary ssDNA. The excitation and emission wavelength were $358 \mathrm{~nm}$ and $461 \mathrm{~nm}$, respectively.

As shown in Fig. 3, the fluorescence of the LAPS surface after the DNA hybridization is much higher than that of the bare surface or after silanization and ssDNA immobilization processes. The region with gold layer on the LAPS surface was used for a negative control, which means no DNA immobilization and hybridization event happened on the gold area. The enhanced fluorescence signal detected in regions with $\mathrm{SiO}_{2}$ surface verifies the existence of dsDNA on the LAPS surface and thus, the successful hybridization of target complementary ssDNA with ssDNA probe immobilized on the LAPS surface.

3.2 Label-free electrical detection of DNA hybridization with LAPS

Fig. 4a shows $I-V$ curves of a $\mathrm{Ta}_{2} \mathrm{O}_{5}$-gate LAPS after the immobilization of probe ssDNA molecules and after the hybridization in a solution with different concentrations of complementary target ssDNA from $10 \mathrm{nM}$ to 1 $\mu \mathrm{M}$. All $I-V$ curves were recorded in the same measurement solution. Mismatch ssDNA was used as a control sequence.

As can be seen from Fig. 4a, with increasing the concentration of complementary target ssDNA, the $I-V$ curves of the LAPS shift to the direction of more negative bias voltages. The concentration-dependent response of the LAPS for a complementary and a mismatch DNA is presented in Fig. 4b. A large voltage shift of $\sim 50$ $\mathrm{mV}$ has been observed already at a complementary ssDNA concentration of $10 \mathrm{nM}$. In contrast, the response of the LAPS biosensor to mismatch ssDNA was very small (a few $\mathrm{mV}$ ) even at a high concentration of $1 \mu \mathrm{M}$, which indicates a good specificity of this biosensor for the specific detection of complementary target ssDNA.

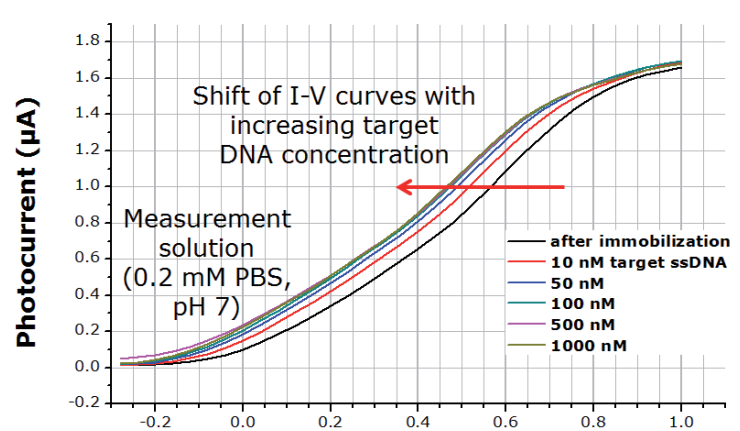

(a)

Bias voltage (V)

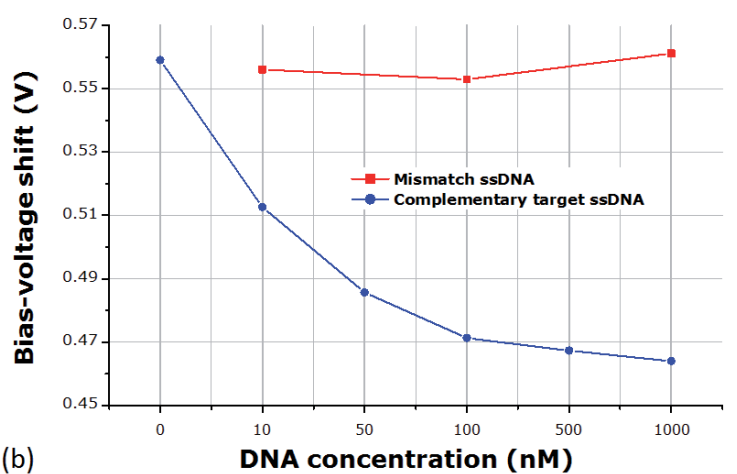

Fig. 4. DNA-hybridization detection with $\mathrm{Ta}_{2} \mathrm{O}_{5}$-gate LAPS: (a) I-V curves after the immobilization of probe ssDNA molecules and after the hybridization in a solution with different concentrations of complementary target ssDNA; (b) bias-voltage shifts vs. concentrations of complementary target and mismatch ssDNA. The measured data were averaged over $n=144$ spots.

\subsection{Mechanisms of signal transduction}

In principle, since the DNA in the solution is negatively charged, the $I-V$ curves of the LAPS chip after the hybridization should shift to the direction of more positive bias voltages. However, in this study, surprisingly, the direction of the DNA-hybridization induced 
voltage shifts was contrary with the results on DNA-hybridization detection with capacitive electrolyte-insulator-semiconductor sensors or transistor structures reported previously $[3,10]$. The possible mechanism that could explain such a behavior is illustrated in Fig. 5 .

We anticipated that the DNA hybridization could lead to DNA conformation changes, resulting in a charge redistribution. In addition, the distance between the DNA molecular charges and the sensor surface is one of the most critical parameters that could strongly affect the expected sensor signal [3]. If flexible coil-like ssDNA molecules lie preferentially flat on the gate surface of the LAPS, they can partially cover the surface active sites for ion interaction as well as prevent potential-determining ions to reach the transducer surface [10]. After hybridization, rigid rod-like dsDNA are formed and the LAPS surface is opened for ion interaction. Thus, the standing up of DNA molecules after hybridization will result in a decrease of negative charges within the Debye length and an ion-concentration redistribution within the intermolecular spaces. All these effects will shift the $I-V$ curve of the LAPS in the direction corresponding to a less negative gatesurface charge that was, in fact, observed in our experiments.

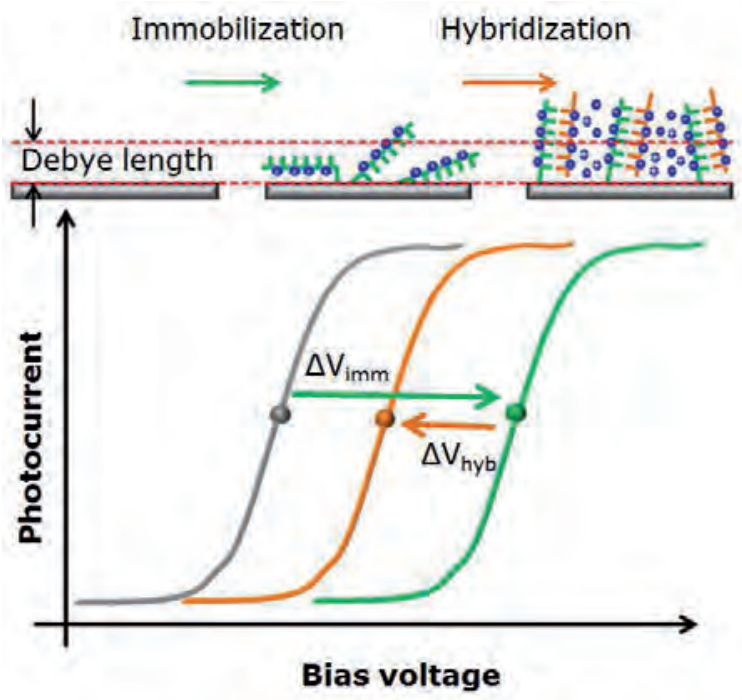

Fig. 5. Mechanism of a label-free electrical detection of DNA hybridization by the intrinsic molecular charge using the LAPS.

\section{Conclusions}

Scanning LAPS was applied for a label-free electrical detection of DNA hybridization. The LAPS signal shows a distinct dependence on the concentration of the complementary target DNA. The mechanism of the DNA-hybridization signal generation has been proposed and discussed. The obtained results demonstrate the feasibility of the scanning LAPS for a labelfree electrical detection of DNA hybridization. The developed LAPS-based DNA biosensor has a great potential as a promising and valuable tool for the DNA detection with a wide range of potential applications.

\section{Acknowledgements}

This work was partially supported by the grants from BMBF (DiaCharge), DAAD (A/11/97272), the National Natural Science Foundation of China (Grant No. 31000448), and the Zhejiang Provincial Natural Science Foundation of China (LY13H180002).

\section{References}

[1] A. Sassolas, B.D. Leca-Bouvier, L.J. Blum, Chemical Reviews 108, 109-139 (2008); doi: $10.1021 / \mathrm{cr} 0684467$

[2] F. Pouthas, C. Gentil, D. Cote, U. Bockelmann, Applied Physics Letters 84,1594-1596 (2004); doi: $10.1063 / 1.1650907$

[3] M.H. Abouzar, A. Poghossian, A.M. Pedraza, D. Gandhi, S. Ingebrandt, W. Moritz, M.J. Schöning. Biosensors and Bioelectronics 26, 3023-3028 (2011); doi: 10.1016/j.bios.2010.12.006

[4] D.G. Hafeman, J.W. Parce, H.M. McConnell, Science 240, 1182-1185 (1988); doi: $10.1126 /$ science.3375810

[5] T. Wagner, C.F. Werner, K. Miyamoto, M.J. Schöning, T. Yoshinobu. Sensors and Actuators B 154, 124-128 (2011); doi: 10.1016/j.snb.2010.03.009

[6] C.F. Werner, A. Mansour, F.M. Rateike, S. Schusser, T. Wagner, T. Yoshinobu, M. Keusgen, M.J. Schöning, 10. Dresdner Sensor-Symposium 43, 277-280 (2011); doi: 10.5162/10dss2011/14.3

[7] J.R. Siqueira, C.F. Werner, M. Bäcker, A. Poghossian, V. Zucolotto, O.N. Oliveira, M.J. Schöning, The Journal of Physical Chemical C 113, 14765-14770 (2009); doi: 10.1021/jp904777t

[8] T. Yoshinobu, H. Ecken, A. Poghossian, A. Simonis, H. Iwasaki, H. Lüth, M.J. Schöning, Electroanalysis 13, 733-736 (2001); doi: 10.1002/1521-4109(200105)

[9] A. Poghossian, A. Baade, H. Emons, M.J. Schöning, Sensors and Actuators B 76, 634-638 (2001); doi: 10.1016/S0925-4005(01)00659-1

[10] A. Poghossian, A. Cherstvy, S. Ingebrandt, A. Offenhäusser, M.J. Schöning, Sensors and Actuators B 111-112, 470-480 (2005); doi: 10.1016/j.snb.2005.03.083 